Research Paper

\title{
Resection versus Resection with Preoperative Transcatheter Arterial Chemoembolization for Resectable Hepatocellular Carcinoma Recurrence
}

\author{
Qiang Tao ${ }^{1,2^{*}}$, Wei He ${ }^{1,2^{*}}$, Binkui Li1, ${ }^{*}$, Yun Zheng1,2, Ruhai Zou1,3, Jingxian Shen ${ }^{1,4}$, Wenwu Liu1,2, \\ Yuanping Zhang ${ }^{1,2}$, Yunfei Yuan ${ }^{1,2}$ \\ 1. State Key Laboratory of Oncology in South China and Collaborative Innovation Center for Cancer Medicine, Sun Yat-Sen University Cancer Center, \\ Guangzhou, China \\ 2. Department of Hepatobiliary Oncology, Sun Yat-Sen University Cancer Center, Guangzhou, China \\ 3. Department of Ultrasound, Sun Yat-Sen University Cancer Center, Guangzhou, China \\ 4. Department of Medical Imaging, Sun Yat-Sen University Cancer Center, Guangzhou, China \\ * These authors contributed equally to this work. \\ $\square$ Corresponding author: Yunfei Yuan, E-mail: yuanyf@mail.sysu.edu.cn \\ (c) Ivyspring International Publisher. This is an open access article distributed under the terms of the Creative Commons Attribution (CC BY-NC) license \\ (https://creativecommons.org/licenses/by-nc/4.0/). See http://ivyspring.com/terms for full terms and conditions.
}

Received: 2018.01.19; Accepted: 2018.03.31; Published: 2018.07.16

\begin{abstract}
The value of preoperative transcatheter arterial chemoembolization (TACE) for patients with recurrent hepatocellular carcinoma $(\mathrm{rHCC})$ after liver resection is uncertain. We aimed to determine its effect on postoperative complication and survival. There were 33 patients who received preoperative TACE and repeated liver resection (TACE-LR) and 119 patients who received repeated liver resection (LR) alone for rHCC. Seventy-eight patients (TACE-LR, 28; LR, 50) were identified by propensity score matching (PSM) analysis for comparison of postoperative complication, disease-free survival (DFS) and overall survival (OS). Univariable and multivariable analyses were used to identify predictors for survival. Before matching, the TACE-LR group had more intraoperative blood loss than the LR group $(P<0.05)$. After matching, the TACE-LR group had more intraoperative blood loss and a longer operation time (Both $\mathrm{P}<$ 0.05). In all and matched patients, both groups had similar postoperative complications rate (TACE-LR, 21.2\%; LR, 7.6\%; $P=0.052$ and TACE-LR, 21.4\%; LR, $12.0 \% ; P=0.435)$, DFS $(P=0.81$ and $P=0.41)$ and OS $(P=0.87$ and $P=0.79)$. Preoperative TACE was not a predictor for DFS and OS in multivariable analyses. Preoperative TACE for resectable $\mathrm{rHCC}$ prolongs operating time and increases intraoperative blood loss without improving survival; thus, it should not be recommended as a routine procedure before repeated resection for patients with $\mathrm{rHCCs}$.
\end{abstract}

Key words: Hepatocellular carcinoma; Recurrence; Liver resection; Transcatheter Arterial Chemoembolization.

\section{Introduction}

Hepatocellular carcinoma (HCC) is the sixth most common cancer worldwide and the third leading cause of cancer-related death [1,2]. Liver resection is a potentially curative treatment. However, the 5 -year recurrence rate after resection is up to $70 \%$ [3]. Due to the high recurrence rate, long-term prognosis after curative resection remains unsatisfactory, and the 5-year overall survival (OS) rate after liver resection is $40-50 \%[4,5]$. Therefore, an appropriate treatment for recurrent HCC (rHCC) after liver resection is the key to improving survival.
Repeat liver resection is superior to TACE and remains the first choice for resectable $\mathrm{rHCC}$, with a 5 -year survival rate of $19.4-56 \%[4,6,7]$. Generally, the rate of repeat liver resection is $10.4 \%$ to $31 \%$ [4, 8-10].

Transcatheter arterial chemoembolization (TACE) is a common treatment for unresectable HCC in which tumor ischemic necrosis is induced through arterial injection of chemotherapeutic drugs and embolizing agents. For patients with resectable primary HCC, preoperative TACE has been shown to offer no benefit and even be associated with worse 
survival, longer operating time and a higher incidence of extrahepatic tumors in patients with incomplete or no tumor necrosis after preoperative TACE[3, 11]. In clinical practice, TACE prior to attempted resection for rHCC is used with the hope that it may improve long-term outcome by reducing the viability of tumor cells or decreasing the size of a previously unresectable recurrence. However, there is no study that assesses the value of preoperative TACE for rHCC [12-16]. We aimed to explore the value of TACE prior to liver resection for patients with $\mathrm{rHCC}$ after initial resection for HCC.

\section{Materials and Methods}

\section{Patients}

From January 2001 to December 2014, 152 patients with $\mathrm{rHCC}$ received repeated liver resection at our center. Among them, 33 patients with rHCC received preoperative TACE and repeated liver resection (TACE-LR) and 119 patients with rHCC received repeated liver resection alone (LR). The following inclusion criteria were used: (1) Curative (R0) hepatic resection for both primary and recurrent HCC as described in previous study [17]; (2) no extrahepatic metastasis; (3) preoperative liver function of Child-Pugh class A; (4) preoperative TACE and repeated liver resection or repeated liver resection alone as the initial treatment for recurrence; (5) all HCCs were confirmed by histological examination. The study was approved by the institutional ethics committee of our center. Written informed consent was obtained.

\section{Diagnosis and Treatment of HCC}

The preoperative diagnosis of primary and recurrent HCC was based on clinical criteria from the AASLD [3]. Histopathological criteria were used to confirm HCC diagnoses after resection. In our center, TACE was performed before repeated liver resection for rHCC in the following situations: (1) Patients with potential intrahepatic metastases (8 cases); (2) to downgrade tumors for curative liver resection (6 cases); (3) insufficient liver function for immediate surgery (10 cases); (4) hoping to improve long-term outcome (9 cases).

\section{TACE and resection procedure}

TACE was performed by interventional radiologists who had more than eight years of experience in interventional therapy at the start of the study. We performed TACE using the Seldinger technique. Under local anesthesia, a catheter was introduced into the proper hepatic artery through the femoral artery. Hepatic angiography was performed to obtain information on the tumor size, number, location, and arterial supply and confirm the patency of the portal vein. Then the chemotherapeutic agents, including carboplatin $300 \mathrm{mg}$, epirubicin $50 \mathrm{mg}$ and mitomycin C $6 \mathrm{mg}$ with an appropriate amount of iodized oil according to the tumor size and number, were injected into the arterial branches. The injection was continued until stasis was confirmed in the feeding artery. The effects of TACE were evaluated based on the comparison of CT/MRI scans before and after TACE. Changes in tumor size after TACE were recorded as enlargement, stationary, $<50 \%$ shrinkage, and $\geq 50 \%$ shrinkage.

The surgeons performing resection had 16-22 years of experience in liver surgery. The surgical plan was developed based on tumor extent and liver function. Preferable anatomical resection was performed. We applied Pringle's maneuver with cycles of clamping and unclamping times of 10 minutes and 5 minutes, respectively, and lowered central venous pressure to $2-4 \mathrm{mmHg}$ during parenchyma dissection to control intraoperative bleeding.

\section{Follow-up}

Postoperative complications were observed for 90 days after the operation and graded using the Clavien-Dindo system [18]. The results were reviewed independently by two of the authors, and any disagreement was resolved by consensus. All patients received routine follow-up. The follow-up recurrence examination after the liver resection included a serum alpha-fetoprotein (AFP) test, a liver function test and abdominal enhanced imaging including CT or MRI. The follow-up visits were performed once every 3-6 months during the first two years and every 6-12 months thereafter. The primary endpoint of this study was OS rate, the secondary endpoint was disease-free survival (DFS) rate, and the tertiary endpoints included side effects of the operation, operating time and blood loss. We defined OS time as the interval between the time recurrent HCC was diagnosed after initial liver resection and the time of death or the last follow-up, and DFS time was defined as the interval between the time recurrent HCC was diagnosed after initial liver resection and the time subsequent tumor recurrence was found. This study was censored on January 1, 2016.

\section{Statistical Analysis}

Propensity score matching (PSM) analysis was used to reduce the bias of treatment selection [19]. We performed PSM via logistic regression to estimate a propensity score for each patient. The following covariates entered the model: etiology, primary tumor differentiation, presence or absence of microvascular 
invasion of primary tumors, recurrent tumor number and interval between initial resection and recurrence. We matched 28 patients in the TACE-LR group with 50 patients in the LR group using the one-to-two nearest neighbor matching algorithm without replacement (caliper $=0.2$ ). For all patients and matched patients, continuous variables between the two treatment groups were compared using Student's t-test or the Mann-Whitney U-test; binary and ordinal categorical variables were compared using the chi-square test and Kruskal-Wallis test, respectively. OS and DFS curves were constructed using the Kaplan-Meier method and compared using the log-rank test. Prognostic factors for OS and DFS were assessed using the Cox proportional hazards model in a stepwise manner (probability for stepwise: Entry 0.05, Removal 0.10). The proportional hazards assumption was verified by the Schoenfeld residuals tests and checked by graphical diagnostics. All statistical analyses were performed using SPSS 20.0 (IBM, Armonk, NY, USA) and PSM for SPSS, version 3.02. For all statistical tests, $\mathrm{P}<0.05$ was considered statistically significant. All $\mathrm{P}$ value calculations were two-sided tests. Key raw data in our study have been uploaded onto the Research Data Deposit public platform (www.researchdata.org.cn) with the approval number as RDDA2018000548.

\section{Results}

\section{The Effects of TACE}

In the TACE-LR group, preoperative TACE was performed once in 29 patients, twice in 3 patients and three times in 1 patient. The mean time from the preoperative TACE to liver resection was 67 days. After TACE, tumors shrunk in 23 patients $(<50 \%$ shrinkage, $11 ; \geq 50 \%$ shrinkage, 12$)$, unchanged in 7 patients and enlarged in 3 patients.

\section{Results before PSM}

The clinicopathologic features of patients in these two groups are summarized in Table 1. The median follow-up times were 28.3 months in the TACE-LR group and 34.3 months in the LR group. Patients in the TACE-LR group were associated with more multiple recurrent $\mathrm{HCC}$ and a shorter interval between initial resection and recurrence when compared with that of patients in the LR group (both $\mathrm{P}<0.05)$. There was no significant difference between the two groups in size of the primary and recurrent tumors, AFP level or prothrombin time (PT). The surgical characteristics and postoperative complications of the two groups are shown in Table 2. The TACE-LR group had more intraoperative blood loss and greater extent of resection than the LR group $(\mathrm{P}=$
0.017 and $P=0.023$, respectively). Both groups showed similar postoperative complication rates and similar operating time. The 1-, 3-, and 5-year OS rates were $92.9 \%, 71.1 \%$, and $63.2 \%$, respectively, for the LR group and $87.9 \%, 58.2 \%$, and $58.2 \%$ for the TACE-LR group. Univariable analyses revealed that gender $(\mathrm{P}=$ 0.011; hazard ratio $[\mathrm{HR}]=2.362 ; 95 \% \mathrm{CI}=1.216$, 4.590), recurrent tumor size $(\mathrm{P}=0.047$; $\mathrm{HR}=1.118$; $95 \% \mathrm{CI}=1.001,1.247)$, primary tumor differentiation $(\mathrm{P}=0.029 ; \mathrm{HR}=1.813 ; 95 \% \mathrm{CI}=1.063,3.090)$ and interval between initial resection and recurrence $(\mathrm{P}=$ $0.012 ; \mathrm{HR}=0.982 ; 95 \% \mathrm{CI}=0.969,0.996)$ were associated with OS rates. The Cox proportional hazards model revealed that the independent predictive factors for OS were recurrent tumor size $(\mathrm{P}$ $=0.004 ; \mathrm{HR}=1.177 ; 95 \% \mathrm{CI}=1.052,1.316)$ and interval between initial resection and recurrence $(\mathrm{P}=0.007$; $\mathrm{HR}=0.980 ; 95 \% \mathrm{CI}=0.966,0.995)$ (Table 3$)$. However, preoperative TACE was not associated with OS rates. The 1-, 3-, and 5-year DFS rates were 70.7\%, 37.1\%, and $28.0 \%$, respectively, for the LR group and $75.2 \%$, $28.5 \%$, and $28.5 \%$ for the TACE-LR group. Univariable analyses revealed that age $(\mathrm{P}=0.048 ; \mathrm{HR}=0.983 ; 95 \%$ $\mathrm{CI}=0.966,1.000)$, gender $(\mathrm{P}=0.038 ; \mathrm{HR}=1.831 ; 95 \%$ $\mathrm{CI}=1.035,3.239)$, recurrent tumor number $(\mathrm{P}=0.020$; $\mathrm{HR}=1.680 ; 95 \% \mathrm{CI}=1.085,2.601)$ and primary tumor differentiation $(\mathrm{P}=0.005 ; \mathrm{HR}=1.767 ; 95 \% \mathrm{CI}=1.183$, 2.639) were important factors for DFS rates. The Cox proportional hazards model demonstrated that the independent predictive factor for DFS was primary tumor differentiation $(\mathrm{P}=0.035 ; \mathrm{HR}=1.567 ; 95 \% \mathrm{CI}=$ 1.033, 2.378) (Table 4). However, preoperative TACE was not shown to also influence DFS rates. Neither the DFS nor OS rate was significantly different between the two groups $(\mathrm{P}=0.809$ and $\mathrm{P}=0.870$, respectively Fig $1 \mathrm{~A} \& \mathrm{~B})$.

\section{Results after PSM}

PSM analysis was performed to reduce the bias of covariates between these two groups (Figure S1). After matching, patients in both groups had similar clinicopathologic features (Table 1). A total of 50 of the 119 patients in the LR group and 28 of the 33 patients in the TACE-LR group were matched for further analyses. The TACE-LR group had significantly more intraoperative blood loss and longer operating time than the LR group $(\mathrm{P}=0.018$ and $\mathrm{P}=0.047$, respectively) (Table 2 ). The type of resection was similar between the two groups $(\mathrm{P}=0.607)$. The overall complication rate was $12.0 \%$ in the LR group, which was not significantly different from the $21.4 \%$ rate observed in the TACE-LR group $(\mathrm{P}=0.435)$. No treatment-related deaths were observed in either group. 
Table 1. Preoperative Clinical Characteristics of Patients Before and After PSM

\begin{tabular}{|c|c|c|c|}
\hline \multirow[t]{2}{*}{ Parameter } & \multicolumn{3}{|l|}{ Before Matching } \\
\hline & LR Group $(n=119)$ & TACE-LR Group $(n=33)$ & SMD \\
\hline Age $(y)$ & $49.00[43.50,57.00]$ & $50.00[42.00,60.00]$ & 0.14 \\
\hline Gender $(\mathrm{M} / \mathrm{F})$ & $109 / 10(91.6 / 8.4)$ & $26 / 7(78.8 / 21.2)$ & 0.367 \\
\hline Liver cirrhosis (no/yes) & $47 / 72(39.5 / 60.5)$ & $11 / 22(33.3 / 66.7)$ & 0.128 \\
\hline Etiology (Non-hepatitis/ HBV \& HCV) & $9 / 110(7.6 / 92.4)$ & $1 / 32(3.0 / 97.0)$ & 0.203 \\
\hline Total bilirubin level $(\mu \mathrm{mol} / \mathrm{L})$ & $13.40[9.86,16.50]$ & $13.10[9.50,17.00]$ & 0.044 \\
\hline Albumin level (g/L) & $42.40[40.40,44.90]$ & $42.60[40.30,44.40]$ & 0.147 \\
\hline PT (s) & $12.05[11.50,12.80]$ & $12.30[11.20,13.70]$ & 0.156 \\
\hline Child-Pugh score $(6 / 5)$ & $10 / 109(8.4 / 91.6)$ & $2 / 31(6.1 / 93.9)$ & 0.167 \\
\hline ALT level (U/L) & $30.10[22.25,43.20]$ & $37.00[24.10,48.80]$ & 0.333 \\
\hline AFP level $(\leq 400 />400 \mathrm{ng} / \mathrm{mL})$ & $96 / 23(80.7 / 19.3)$ & $28 / 5(84.8 / 15.2)$ & 0.111 \\
\hline Primary tumor number $(1 />1)$ & $96 / 23(80.7 / 19.3)$ & $26 / 7(78.8 / 21.2)$ & 0.047 \\
\hline Primary tumor size $(\mathrm{cm})$ & $5.00[3.50,8.00]$ & $4.90[3.00,6.50]$ & 0.176 \\
\hline Microvascular invasion of primary tumor (no/yes) & $109 / 10(91.6 / 8.4)$ & $32 / 1(97.0 / 3.0)$ & 0.233 \\
\hline Primary tumor differentiation (poor/moderate \& well) & $84 / 35(70.6 / 29.4)$ & $18 / 15(54.5 / 45.5)$ & 0.336 \\
\hline Recurrent tumor size $(\mathrm{cm})$ & $3.00[2.50,4.50]$ & $3.00[2.30,5.00]$ & 0.081 \\
\hline Recurrent tumor number $(1 />1)$ & $98 / 21(82.4 / 17.6)$ & $18 / 15(54.5 / 45.5)$ & 0.627 \\
\hline Interval between initial resection and recurrence (month) & $27.14[14.44,47.29]$ & $20.11[10.35,31.41]$ & 0.287 \\
\hline \multirow[t]{2}{*}{ Parameter } & \multicolumn{3}{|l|}{ After Matching } \\
\hline & LR Group $(n=50)$ & TACE-LR Group $(\mathrm{n}=28)$ & SMD \\
\hline Age $(y)$ & $51.00[38.75,57.00]$ & $47.00[41.25,61.25]$ & 0.045 \\
\hline Gender $(\mathrm{M} / \mathrm{F})$ & $44 / 6(88.0 / 12.0)$ & $21 / 7(75.0 / 25.0)$ & 0.34 \\
\hline Liver cirrhosis (no/yes) & $19 / 31(38.0 / 62.0)$ & $10 / 18(35.7 / 64.3)$ & 0.047 \\
\hline Etiology (Non-hepatitis/ HBV \& HCV) & $5 / 45(10.0 / 90.0)$ & $1 / 27(3.6 / 96.4)$ & 0.258 \\
\hline Total bilirubin level $(\mu \mathrm{mol} / \mathrm{L})$ & $13.50[10.95,15.97]$ & $12.30[9.32,16.85]$ & 0.077 \\
\hline Albumin level (g/L) & $42.45[40.42,44.98]$ & $43.20[40.30,44.73]$ & 0.091 \\
\hline PT (s) & $12.20[11.60,13.45]$ & $11.50[11.07,13.15]$ & 0.154 \\
\hline Child-Pugh score $(6 / 5)$ & 6/44 (12.0/88.0) & $2 / 31(7.1 / 92.9)$ & 0.185 \\
\hline ALT level (U/L) & $28.20[18.62,37.83]$ & $36.50[22.70,43.25]$ & 0.341 \\
\hline AFP level $(\leq 400 />400 \mathrm{ng} / \mathrm{mL})$ & $40 / 10(80.0 / 20.0)$ & $24 / 4(85.7 / 14.3)$ & 0.152 \\
\hline Primary tumor number $(1 />1)$ & $39 / 11(78.0 / 22.0)$ & $22 / 6(78.6 / 21.4)$ & 0.014 \\
\hline Primary tumor size $(\mathrm{cm})$ & $5.75[4.00,8.00]$ & $4.85[3.00,6.50]$ & 0.288 \\
\hline Microvascular invasion of primary tumor (no/yes) & $48 / 2(96.0 / 4.0)$ & $27 / 1(96.4 / 3.6)$ & 0.022 \\
\hline Primary tumor differentiation (poor/ moderate \& well) & $28 / 22(56.0 / 44.0)$ & $17 / 11(60.7 / 39.3)$ & 0.096 \\
\hline Recurrent tumor size $(\mathrm{cm})$ & $3.00[2.50,4.38]$ & $3.00[2.27,4.25]$ & 0.085 \\
\hline Recurrent tumor number $(1 />1)$ & $33 / 17(66.0 / 34.0)$ & $18 / 10(64.3 / 35.7)$ & 0.036 \\
\hline Interval between initial resection and recurrence (month) & $24.02[12.39,40.44]$ & $22.49[9.14,31.31]$ & 0.08 \\
\hline
\end{tabular}

Values are presented as the median (IQR) or $\mathrm{n}(\%)$.

SMD, standardized mean difference; HBV, hepatitis B virus; $\mathrm{HCV}$, hepatitis C virus; PT, prothrombin time; ALT, alanine aminotransferase; AFP, alpha-fetoprotein.

Table 2. Comparison of Perioperative Characteristics Between Groups Before and After Matching

\begin{tabular}{|c|c|c|c|c|c|c|}
\hline \multirow[t]{2}{*}{ Variable } & \multicolumn{4}{|l|}{ Before Matching } & \multicolumn{2}{|l|}{ After Matching } \\
\hline & TACE-LR Group $(n=33)$ & LR Group $(\mathrm{n}=119)$ & P Value & TACE-LR Group $(\mathrm{n}=28)$ & LR Group $(n=50)$ & P Value \\
\hline Operating time (minute) & $216.5 \pm 99.2$ & $184.3 \pm 57.7$ & 0.155 & $217.1 \pm 103.4$ & $177.6 \pm 46.8$ & 0.047 \\
\hline Blood loss (ml) & $630.3 \pm 882.0$ & $350.8 \pm 385.8$ & 0.017 & $623.7 \pm 802.3$ & $305.2 \pm 198.6$ & 0.018 \\
\hline Hepatic portal blocking (no/yes) & $17 / 16(51.5 / 48.5)$ & $83 / 36(69.7 / 30.3)$ & 0.051 & $15 / 13(53.6 / 46.4)$ & $34 / 16(68.0 / 32.0)$ & 0.206 \\
\hline Type of resection & & & 0.023 & & & 0.607 \\
\hline$<1$ segmentectomy & $12(36.4 \%)$ & $60(50.4 \%)$ & & $12(42.9 \%)$ & $23(46.0 \%)$ & \\
\hline 1-2 segmentectomy & $12(36.4 \%)$ & $48(40.3 \%)$ & & $11(39.3 \%)$ & $22(44.0 \%)$ & \\
\hline$>2$ segmentectomy & $9(27.3 \%)$ & $11(9.2 \%)$ & & $5(17.9 \%)$ & $5(10.0 \%)$ & \\
\hline Complication & $7(21.2 \%)$ & $9(7.6 \%)$ & 0.052 & $6(21.4 \%)$ & $6(12.0 \%)$ & 0.435 \\
\hline Grade 1 & $4(12.1 \%)$ & $4(3.4 \%)$ & & $3(10.7 \%)$ & $3(6.0 \%)$ & \\
\hline Grade 2 & $2(6.1 \%)$ & $4(3.4 \%)$ & & $2(7.1 \%)$ & $2(4.0 \%)$ & \\
\hline Grade 3 & $0(0.0 \%)$ & $1(0.8 \%)$ & & $0(0.0 \%)$ & $1(2.0 \%)$ & \\
\hline Grade 4 & $1(3.0 \%)$ & $0(0.0 \%)$ & & $1(3.6 \%)$ & $0(0.0 \%)$ & \\
\hline Grade 5 & $0(0.0 \%)$ & $0(0.0 \%)$ & & $0(0.0 \%)$ & $0(0.0 \%)$ & \\
\hline
\end{tabular}

The 1-, 3-, and 5-year OS rates were 93.8\%, 68.0\%, and $61.9 \%$, respectively, in the LR group and $85.7 \%$, $60.6 \%$, and $60.6 \%$ in the TACE-LR group. The 1-, 3-, and 5-year DFS rates were $63.1 \%, 26.6 \%$, and $18.7 \%$, respectively, in the LR group and $70.6 \%, 37.2 \%$, and $37.2 \%$ in the TACE-LR group. Both groups had similar DFS and $O S$ rates. $(P=0.407$ and $P=0.791$, respectively) (Fig 1 C\&D). The Cox proportional hazards model revealed that the independent predictive factors for OS were primary tumor differentiation $(\mathrm{P}=0.040 ; \mathrm{HR}=2.198 ; 95 \% \mathrm{CI}=1.038$, 4.651) and interval between initial resection and recurrence $(\mathrm{P}=0.044 ; \mathrm{HR}=0.975 ; 95 \% \mathrm{CI}=0.951$, 0.999). The Cox proportional hazards model demonstrated that the independent predictive factors for DFS was primary tumor differentiation $(\mathrm{P}=0.012$; $\mathrm{HR}=2.025 ; 95 \% \mathrm{CI}=1.171,3.505)$. (Table S1\&S2) Preoperative TACE was not shown to influence OS and DFS rates after matching. 
Table 3. Univariable and Multivariable Analysis of Predictors for OS

\begin{tabular}{|c|c|c|c|c|c|c|}
\hline \multirow[t]{2}{*}{ Variable } & \multicolumn{3}{|c|}{ Univariable Analysis } & \multicolumn{3}{|c|}{ Multivariable Analysis } \\
\hline & $\overline{\mathrm{HR}}$ & $95 \% \mathrm{CI}$ & P Value & HR & $95 \% \mathrm{CI}$ & P Value \\
\hline Pre-operative TACE (no/yes) & 0.946 & $0.487-1.839$ & 0.870 & 0.631 & $0.311-1.281$ & 0.203 \\
\hline Age $(y)$ & 0.997 & $0.975-1.019$ & 0.763 & & & \\
\hline Gender (M/F) & 2.362 & $1.216-4.590$ & 0.011 & 1.870 & $0.920-3.799$ & 0.084 \\
\hline Liver cirrhosis (no/yes) & 1.366 & $0.771-2.421$ & 0.285 & & & \\
\hline Etiology (Non-hepatitis/HBV \& HCV) & 0.612 & $0.261-1.434$ & 0.258 & & & \\
\hline Total bilirubin level $(\mu \mathrm{mol} / \mathrm{L})$ & 1.033 & $0.975-1.093$ & 0.270 & & & \\
\hline Albumin level (g/L) & 0.997 & $0.926-1.073$ & 0.938 & & & \\
\hline PT (s) & 1.171 & $0.999-1.373$ & 0.052 & & & \\
\hline Child-Pugh score $(6 / 5)$ & 1.17 & $0.465-2.950$ & 0.738 & & & \\
\hline ALT level (U/L) & 1.002 & $0.992-1.013$ & 0.652 & & & \\
\hline AFP level $(\leq 400 />400 \mathrm{ng} / \mathrm{mL})$ & 1.347 & $0.710-2.557$ & 0.362 & & & \\
\hline Primary tumor number $(1 />1)$ & 0.841 & $0.424-1.670$ & 0.621 & & & \\
\hline Primary tumor size $(\mathrm{cm})$ & 1.026 & $0.945-1.115$ & 0.537 & & & \\
\hline Microvascular invasion of primary tumor (no/yes) & 1.093 & $0.393-3.042$ & 0.865 & & & \\
\hline Primary tumor differentiation (moderate \& well/poor) & 1.813 & $1.063-3.090$ & 0.029 & 1.740 & $0.993-3.051$ & 0.053 \\
\hline Recurrent tumor size $(\mathrm{cm})$ & 1.118 & $1.001-1.247$ & 0.047 & 1.177 & $1.052-1.316$ & 0.004 \\
\hline Recurrent tumor number $(1 />1)$ & 1.210 & $0.659-2.224$ & 0.539 & & & \\
\hline Interval between initial resection and recurrence (month) & 0.982 & $0.969-0.996$ & 0.012 & 0.980 & $0.966-0.995$ & 0.007 \\
\hline
\end{tabular}

TACE, transcatheter arterial chemoembolization; $\mathrm{HBV}$, hepatitis $\mathrm{B}$ virus; $\mathrm{HCV}$, hepatitis $\mathrm{C}$ virus; PT, prothrombin time; ALT, alanine aminotransferase; AFP, alpha-fetoprotein.

Table 4. Univariable and Multivariable Analysis of Predictors for DFS

\begin{tabular}{|c|c|c|c|c|c|c|}
\hline \multirow[t]{2}{*}{ Variable } & \multicolumn{3}{|c|}{ Univariable Analysis } & \multicolumn{3}{|c|}{ Multivariable Analysis } \\
\hline & HR & $95 \% \mathrm{CI}$ & P Value & HR & $95 \% \mathrm{CI}$ & P Value \\
\hline Pre-operative TACE (no/yes) & 1.060 & $0.660-1.704$ & 0.809 & 0.865 & $0.525-1.426$ & 0.570 \\
\hline Age $(y)$ & 0.983 & $0.966-1.000$ & 0.048 & 0.989 & $0.971-1.007$ & 0.218 \\
\hline Gender (M/F) & 1.831 & $1.035-3.239$ & 0.038 & 1.540 & $0.847-2.799$ & 0.157 \\
\hline Liver cirrhosis (no/yes) & 1.357 & $0.887-2.074$ & 0.159 & & & \\
\hline Etiology (Non-hepatitis/HBV \& HCV) & 0.835 & $0.387-1.802$ & 0.646 & & & \\
\hline Total bilirubin level ( $\mu \mathrm{mol} / \mathrm{L})$ & 1.007 & $0.965-1.051$ & 0.748 & & & \\
\hline Albumin level (g/L) & 0.987 & $0.932-1.045$ & 0.657 & & & \\
\hline $\mathrm{PT}(\mathrm{s})$ & 1.119 & $0.987-1.270$ & 0.079 & & & \\
\hline Child-Pugh score $(6 / 5)$ & 0.580 & $0.137-2.450$ & 0.459 & & & \\
\hline ALT level (U/L) & 0.997 & $0.989-1.005$ & 0.423 & & & \\
\hline AFP level $(\leq 400 />400 \mathrm{ng} / \mathrm{mL})$ & 1.332 & $0.805-2.203$ & 0.264 & & & \\
\hline Primary tumor number $(1 />1)$ & 0.821 & $0.503-1.343$ & 0.433 & & & \\
\hline Primary tumor size $(\mathrm{cm})$ & 0.986 & $0.927-1.049$ & 0.659 & & & \\
\hline Microvascular invasion of primary tumor (no/yes) & 1.185 & $0.548-2.561$ & 0.666 & & & \\
\hline Primary tumor differentiation (moderate \& well/ poor) & 1.767 & $1.183-2.639$ & 0.005 & 1.567 & $1.033-2.378$ & 0.035 \\
\hline Recurrent tumor size $(\mathrm{cm})$ & 1.045 & $0.960-1.137$ & 0.311 & & & \\
\hline Recurrent tumor number $(1 />1)$ & 1.680 & $1.085-2.601$ & 0.020 & 1.553 & $0.980-2.460$ & 0.061 \\
\hline Interval between initial resection and recurrence (month) & 0.995 & $0.987-1.002$ & 0.172 & & & \\
\hline
\end{tabular}

TACE, transcatheter arterial chemoembolization; HBV, hepatitis B virus; HCV, hepatitis C virus; PT, prothrombin time; ALT, alanine aminotransferase; AFP,

alpha-fetoprotein.

\section{Subsequent Tumor Recurrence and Treatment}

During the follow-up period after hepatectomy for recurrent tumors, there were 22 and 80 subsequent recurrent cases in the TACE-LR group and the LR group, respectively. Treatments for subsequent recurrence in the TACE-LR group were TACE (12 patients), liver resection (4 patients), ablation (5 patients) and supportive treatment (1 patient). Treatments for subsequent recurrence in the LR group included TACE (43 patients), liver resection (17 patients), ablation (15 patients), sorafenib (2 patients) and supportive treatment ( 3 patients).

\section{Discussion}

Recurrence of HCC is recognized as an important prognostic factor for patients after curative liver resection for HCC. Therefore, treatment of recurrent HCC is the key to improving survival. Repeat hepatectomy is the most accepted treatment for recurrent HCC $[4,6]$. A recent study also found that resection is superior to TACE for recurrent HCC after initial resection [7].

Although TACE was initially established as a palliative treatment for unresectable HCC [20]. In recent years, it has also been performed preoperatively on patients with resectable HCC. The main purposes of preoperative TACE were to improve the detection rate of latent intrahepatic metastatic foci; to increase the resectability rate of HCC sy down-staging tumors that are either initially borderline resectable or unresectable; to provide sufficient time for therapy in cases of damaged liver function; and finally, to improve OS and DFS rates 
A

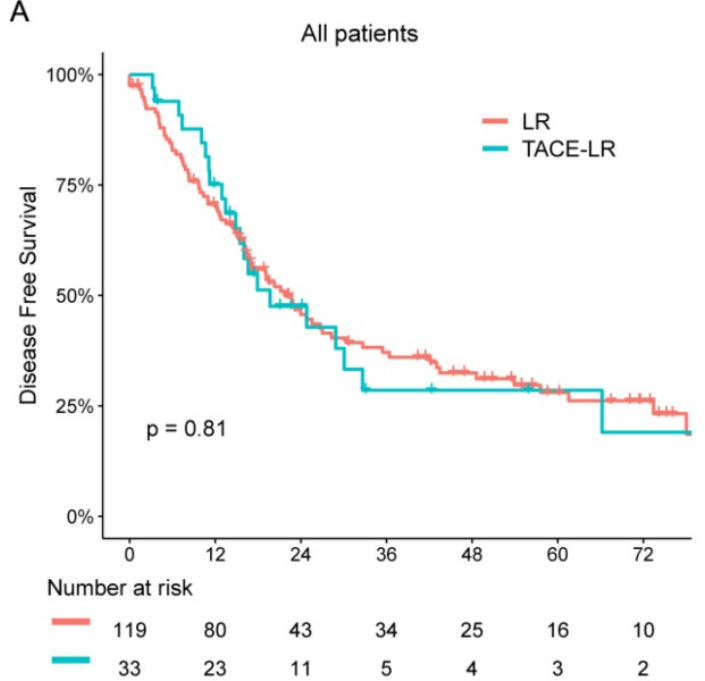

C

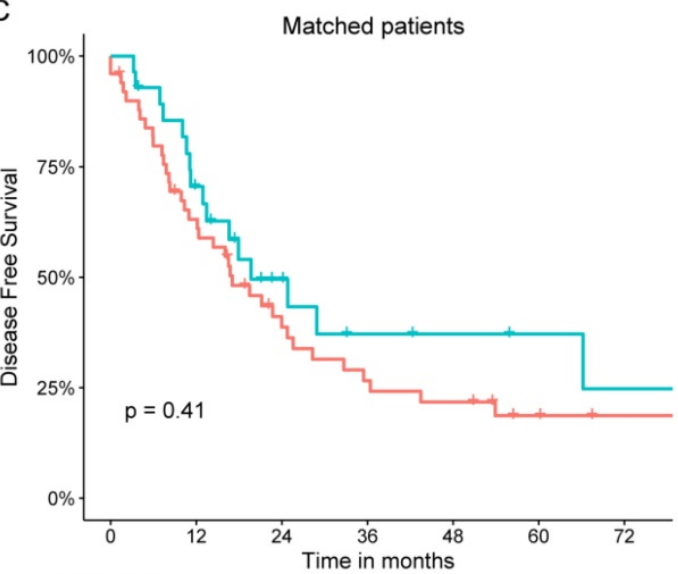

Number at risk

$\begin{array}{rrrrrrr}50 & 30 & 16 & 11 & 9 & 5 & 3 \\ -\quad 28 & 18 & 9 & 5 & 4 & 3 & 2\end{array}$
B

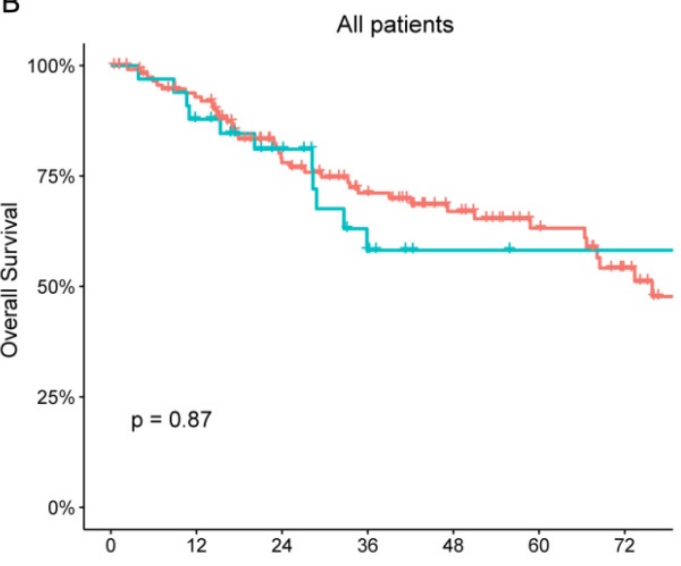

Number at risk

$\begin{array}{lllllll}119 & 102 & 73 & 58 & 43 & 30 & 19\end{array}$

D

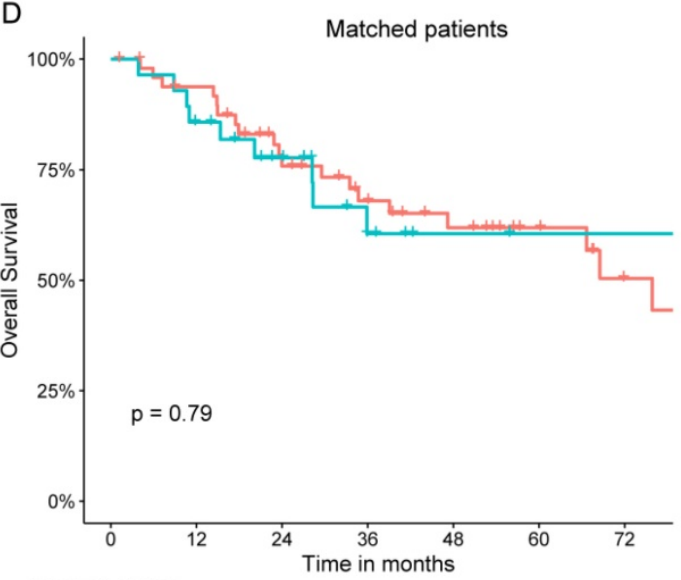

Number at risk

$\begin{array}{rrrrrrr}50 & 44 & 32 & 25 & 19 & 13 & 7 \\ -\quad 28 & 23 & 17 & 9 & 6 & 5 & 5\end{array}$

Figure 1. OS and DFS of patients with recurrent HCC. (A) and (B), DFS and OS of patients receiving liver resection and preoperative TACE for recurrent HCC before PSM. (C) and (D), DFS and OS of patients receiving liver resection and preoperative TACE for recurrent HCC after PSM.

after curative resection [20, 21]. However, for patients with resectable primary HCC, preoperative TACE is proven to be associated with worse survival, longer operating time and higher rates of extrahepatic tumors. Thus, TACE prior to resection for primary HCC offers no benefit and is not recommended by AASLD [3, 22]. However, for patients with resectable recurrent $\mathrm{HCC}$, there is no study to evaluate the value of preoperative TACE. In this study, we compared the effects of TACE followed by liver resection with those of liver resection alone in the treatment of resectable recurrent HCC by using PSM analysis.

In our study, we found longer operating time and more intraoperative blood loss in the TACE-LR group than the LR group. This result is similar to previous studies on the effect of TACE prior to liver resection for patients with primary HCC. These studies have shown that preoperative TACE increases difficulty in hepatic parenchyma dissection due to inflammatory pedicles, perihepatic adhesions, or arterial thrombosis caused by TACE, which result in longer operating time and more intraoperative bleeding [23, 24]. The difficulty of repeated live resection for recurrent HCC is significantly increased due to postoperative inflammatory reaction and perihepatic adhesions after initial resection for primary HCC. Additional TACE prior to repeated resection will aggravate these issues. As a result, preoperative TACE for recurrent HCC should be performed prudently due to increased surgical difficulty and risk. Moreover, postoperative complications should also be considered before making the decision regarding recurrence treatment. We found no significant difference in the postoperative complications after repeated liver resection for recurrent HCC between two groups. This finding is in line with previous research on the postoperative results in patients with primary HCC [16]. 
Other than short-term outcomes, the DFS and OS should be considered when making a treatment choice. In our study, no significant differences were observed between these two groups in DFS and OS rates. In multivariable analyses, preoperative TACE was not a prognostic factor for DFS and OS. Prognostic factors were recurrent tumor number, primary tumor differentiation, recurrent tumor size, and the interval between initial resection and recurrence, which suggests that the role of primary and recurrent tumor characteristics are more important than treatment modality. Previous studies presented similar results in patients with resectable primary HCC, showing that preoperative TACE did not improve the DFS or OS [22,25]. They suggested that preoperative TACE should only be performed in patients with borderline resectability to make it possible to perform curative resection.

Several studies on primary HCC showed that preoperative TACE reduced tumor recurrence and improved survival rate in patients with large tumors [21, 26], advanced HCC [27], and severe liver dysfunction [28]. In our study, compared with patients in the LR group, patients in the TACE-LR group were associated with a larger proportion of multiple recurrent HCC and a shorter interval between initial resection and recurrence. This finding suggests that healthcare providers tend to perform TACE prior to repeated resection on patients with aggressive recurrence such as early, large and multiple recurrent tumors. To better compare the long-term outcome of preoperative TACE, we used PSM to adjust potential confounding factors and to reduce the bias of treatment selection between the two groups. Still, we found that preoperative TACE does not benefit patients with recurrent HCC after repeated live resection.

To our knowledge, there is a lack of data on the role of TACE prior to repeated resection for recurrent HCC after liver resection. Therefore, the results of our study, which were obtained in matched patients with similar clinical characteristics between the TACE-LR and LR groups, can provide valuable information in guiding the management of resectable recurrent HCC after liver resection.

Our study has several limitations. First, this is a single-center retrospective study with a relatively small number of patients. Thus, multi-center studies will be necessary to validate our conclusions. In addition, more than $90 \%$ of the included HCCs were caused by HBV infection. Therefore, it may be difficult to generalize our results to those of HCCs caused by HCV infection or alcohol use.

\section{Conclusions}

In conclusion, preoperative TACE for resectable recurrent $\mathrm{HCC}$ prolongs operating time and increases intraoperative blood loss without improving the DFS and OS; thus, it should not be recommended as a routine procedure before repeated resection for patients with recurrent HCC.

\section{Abbreviations}

HCC: hepatocellular carcinoma; rHCC: recurrent HCC; TACE: transcatheter arterial chemoembolization; PSM: propensity score matching; TACE-LR: preoperative TACE and repeated liver resection; LR: repeated liver resection; AASLD: American Association for the Study of Liver Diseases; AFP: alpha-fetoprotein; $\mathrm{HCV}$ : hepatitis $\mathrm{C}$ virus; OS: overall survival; DFS: disease-free survival; MDT: multidisciplinary team; US: ultrasound; ALB: albumin; TBIL: total bilirubin; ALT: alanine aminotransferase.

\section{Supplementary Material}

Supplementary figure and tables. http://www.jcancer.org/v09p2778s1.pdf

\section{Acknowledgements}

This work was supported by grants from the National Natural science Foundation of China (No. $81772598,81772625)$ and the Sun Yat-Sen University Clinical Research 5010 Program (No. 2012010).

\section{Availability of data and materials}

The key raw data have been deposited into the Research Data Deposit (www.researchdata.org.cn), with the approval number of RDDA2018000548.

\section{Competing Interests}

The authors have declared that no competing interest exists.

\section{References}

1. Siegel RL, Miller KD, Jemal A. Cancer statistics, 2016. CA: a cancer journal for clinicians. 2016; 66: 7-30.

2. Chen $W$, Zheng R, Baade PD, Zhang S, Zeng H, Bray F, et al. Cancer statistics in China, 2015. CA: a cancer journal for clinicians. 2016; 66: 115-32.

3. Bruix J, Sherman M, American Association for the Study of Liver D. Management of hepatocellular carcinoma: an update. Hepatology. 2011; 53: 1020-2.

4. Poon RT, Fan ST, Lo CM, Liu CL, Wong J. Intrahepatic recurrence after curative resection of hepatocellular carcinoma: long-term results of treatment and prognostic factors. Annals of surgery. 1999; 229: 216-22.

5. Ikai I, Arii S, Kojiro M, Ichida T, Makuuchi M, Matsuyama Y, et al. Reevaluation of prognostic factors for survival after liver resection in patients with hepatocellular carcinoma in a Japanese nationwide survey. Cancer. 2004; 101: 796-802.

6. Shimada M, Takenaka K, Gion T, Fujiwara Y, Kajiyama K, Maeda T, et al. Prognosis of recurrent hepatocellular carcinoma: a 10-year surgical experience in Japan. Gastroenterology. 1996; 111: 720-6.

7. Wang DY, Liu L, Qi XS, Su CP, Chen X, Liu X, et al. Hepatic Re-resection Versus Transarterial Chemoembolization for the Treatment of Recurrent Hepatocellular Carcinoma after Initial Resection: a Systematic Review and 
Meta-analysis. Asian Pacific journal of cancer prevention : APJCP. 2015; 16: 5573-8.

8. Suenaga M, Sugiura H, Kokuba Y, Uehara S, Kurumiya T. Repeated hepatic resection for recurrent hepatocellular carcinoma in eighteen cases. Surgery. 1994; 115: 452-7.

9. Kakazu T, Makuuchi M, Kawasaki S, Miyagawa S, Hashikura Y, Kosuge T, et al. Repeat hepatic resection for recurrent hepatocellular carcinoma. Hepato-gastroenterology. 1993; 40: 337-41.

10. Minagawa M, Makuuchi M, Takayama T, Kokudo N. Selection criteria for repeat hepatectomy in patients with recurrent hepatocellular carcinoma. Annals of surgery. 2003; 238: 703-10.

11. Qi X, Liu L, Wang D, Li H, Su C, Guo X. Hepatic resection alone versus in combination with pre- and post-operative transarterial chemoembolization for the treatment of hepatocellular carcinoma: A systematic review and meta-analysis. Oncotarget. 2015; 6: 36838-59.

12. Uchida M, Kohno H, Kubota H, Hayashi T, Yamanoi A, Kimoto T, et al. Role of preoperative transcatheter arterial oily chemoembolization for resectable hepatocellular carcinoma. World journal of surgery. 1996; 20: 326-31.

13. Adachi E, Matsumata T, Nishizaki T, Hashimoto H, Tsuneyoshi M, Sugimachi K. Effects of preoperative transcatheter hepatic arterial chemoembolization for hepatocellular carcinoma. The relationship between postoperative course and tumor necrosis. Cancer. 1993; 72: 3593-8.

14. Lee KT, Lu YW, Wang SN, Chen HY, Chuang SC, Chang WT, et al. The effect of preoperative transarterial chemoembolization of resectable hepatocellular carcinoma on clinical and economic outcomes. J Surg Oncol. 2009; 99: 343-50.

15. Wu CC, Ho YZ, Ho WL, Wu TC, Liu TJ, P'Eng F K. Preoperative transcatheter arterial chemoembolization for resectable large hepatocellular carcinoma: a reappraisal. The British journal of surgery. 1995; 82: 122-6.

16. Zhou Y, Zhang X, Wu L, Ye F, Su X, Shi L, et al. Meta-analysis: preoperative transcatheter arterial chemoembolization does not improve prognosis of patients with resectable hepatocellular carcinoma. BMC gastroenterology. 2013; 13: 51.

17. Poon RT, Fan ST, Ng IO, Lo CM, Liu CL, Wong J. Different risk factors and prognosis for early and late intrahepatic recurrence after resection of hepatocellular carcinoma. Cancer. 2000; 89: 500-7.

18. Clavien PA, Barkun J, de Oliveira ML, Vauthey JN, Dindo D, Schulick RD, et al. The Clavien-Dindo classification of surgical complications: five-year experience. Annals of surgery. 2009; 250: 187-96.

19. Ho DE, Imai K, King G, Stuart EA. Matching as nonparametric preprocessing for reducing model dependence in parametric causal inference. Polit Anal. 2007; 15: 199-236.

20. Choi GH, Kim DH, Kang CM, Kim KS, Choi JS, Lee WJ, et al. Is preoperative transarterial chemoembolization needed for a resectable hepatocellular carcinoma? World J Surg. 2007; 31: 2370-7.

21. Lu CD, Peng SY, Jiang XC, Chiba Y, Tanigawa N. Preoperative transcatheter arterial chemoembolization and prognosis of patients with hepatocellular carcinomas: retrospective analysis of 120 cases. World J Surg. 1999; 23: 293-300.

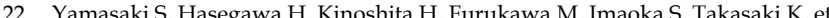
al. A prospective randomized trial of the preventive effect of pre-operative transcatheter arterial embolization against recurrence of hepatocellular carcinoma. Japanese journal of cancer research : Gann. 1996; 87: 206-11.

23. Paye $F$, Jagot $P$, Vilgrain $V$, Farges $O$, Borie D, Belghiti J. Preoperative chemoembolization of hepatocellular carcinoma: a comparative study. Archives of surgery. 1998; 133: 767-72.

24. Luo $\mathrm{YQ}$ Wang $\mathrm{Y}$, Chen $\mathrm{H}, \mathrm{Wu} \mathrm{MC}$. Influence of preoperative transcatheter arterial chemoembolization on liver resection in patients with resectable hepatocellular carcinoma. Hepatobiliary \& pancreatic diseases international : HBPD INT. 2002; 1: 523-6.

25. Harada $\mathrm{T}$, Matsuo $\mathrm{K}$, Inoue $\mathrm{T}$, Tamesue $\mathrm{S}$, Inoue $\mathrm{T}$, Nakamura $\mathrm{H}$. Is preoperative hepatic arterial chemoembolization safe and effective for hepatocellular carcinoma? Annals of surgery. 1996; 224: 4-9.

26. Chen XP, Hu DY, Zhang ZW, Zhang BX, Chen YF, Zhang WG, et al. Role of mesohepatectomy with or without transcatheter arterial chemoembolization for large centrally located hepatocellular carcinoma. Digestive surgery. 2007; 24: 208-13.

27. Sugo H, Futagawa S, Beppu T, Fukasawa M, Kojima K. Role of preoperative transcatheter arterial chemoembolization for resectable hepatocellular carcinoma: relation between postoperative course and the pattern of tumor recurrence. World J Surg. 2003; 27: 1295-9.

28. Kaibori M, Tanigawa N, Matsui $Y$, Saito T, Uchida $Y$, Ishizaki M, et al. Influence of transcatheter arterial chemoembolization on the prognosis after hepatectomy for hepatocellular carcinoma in patients with severe liver dysfunction. Anticancer research. 2006; 26: 3685-92. 